\title{
A novel and efficient surgical knotting technique for high-tension closures
}

\author{
Yongyun Chang ${ }^{1,2 \#}$, Degang Yu ${ }^{1 \#}$, Liao Wang ${ }^{1}$, Jingwei Zhang ${ }^{1}$, Mengning Yan $^{1}$, Huiwu Li ${ }^{1}$, \\ Yuanqing $\mathrm{Mao}^{1}$, Zanjing $\mathrm{Zhai}^{1}$
}

${ }^{1}$ Shanghai Key Laboratory of Orthopaedic Implants, Department of Orthopaedic Surgery, Shanghai Ninth People's Hospital, Shanghai Jiao Tong University School of Medicine, Shanghai, China; ${ }^{2}$ Department of Orthopaedic Surgery, Renji Hospital, School of Medicine, Shanghai Jiao Tong University, Shanghai, China

Contributions: (I) Conception and design: Y Mao, Z Zhai; (II) Administrative support: H Li; (III) Provision of study materials or patients: M Yan; (IV) Collection and assembly of data: L Wang, J Zhang; (V) Data analysis and interpretation: Y Chang, D Yu; (VI) Manuscript writing: All authors; (VII) Final approval of manuscript: All authors.

\#These authors contributed equally to this work as co-first authors.

Correspondence to: Zanjing Zhai; Yuanqing Mao. Shanghai Key Laboratory of Orthopaedic Implants, Department of Orthopaedic Surgery, Shanghai Ninth People’s Hospital, Shanghai Jiao Tong University School of Medicine, Shanghai, China. Email: zanjing_zhai@163.com; yuanqingmao@163.com.

Background: The closure of high-tension incisions without any assistance can be difficult and challenging for surgeons. A common practice is to fix the first knot with a clamp and then tie a reverse locking knot; however, this practice has certain disadvantages. The aim of this study was to introduce a novel and efficient surgical knotting technique with various advantages.

Methods: The two knotting methods used in this study were the absorbable braided suture where the first suture was fixed with a clamp (with assistance) and the SH-9Hospital knotting technique (without assistance) applied on the smooth surface of a cylinder. Mechanical testing was performed using a universal material testing machine. The load-elongation curve and ultimate tensile load (UTL) were recorded.

Results: The mean knotting time was $36.40 \pm 1.50 \mathrm{~s}$ (range, $32-41 \mathrm{~s}$ ) and $24.80 \pm 1.16 \mathrm{~s}$ (range, $21-28 \mathrm{~s}$ ) in the clamp and SH-9Hosptial groups, respectively. The mean UTL was $120.8 \pm 10.14 \mathrm{~N}$ (range, 81.11-136.55 N) and $126.5 \pm 6.29 \mathrm{~N}$ (range, 104.88-139.56 N) in the clamp and SH-9Hospital groups, respectively. The knot strength of the SH-9Hospital technique was not inferior to traditional clinical practice.

Conclusions: The SH-9Hospital knotting technique was a secure, convenient, and efficient method for high-tension closure.

Keywords: Orthopaedic procedures; surgical clamps; suture technique; tensile strength; wound closure

Submitted Sep 08, 2020. Accepted for publication Dec 18, 2020.

doi: 10.21037/atm-20-6332

View this article at: http://dx.doi.org/10.21037/atm-20-6332

\section{Introduction}

Tight and strong incision closures are crucial to orthopaedic operations, especially considering the need for early mobilization and rehabilitation exercises $(1,2)$. Knots slippage or unravelling can result in serious complications and decrease patient satisfaction (3). In orthopaedic procedures, the closure of the joint capsule, fascia, and other soft tissue is often performed under tension, and the first suture loop is always prone to loosening because of tissue tension. A common clinical practice is that the assistant holds the knotted suture with the surgical clamp following the first throw to prevent knot's loosening while the subsequent knot throws are performed (4). However, this usual practice has certain deficiencies. First, clamp fixation to prevent unfolding of the first suture knot may damage the suture and decrease its tensile strength (5-8). Second, the first suture knot may slide leading to a small 

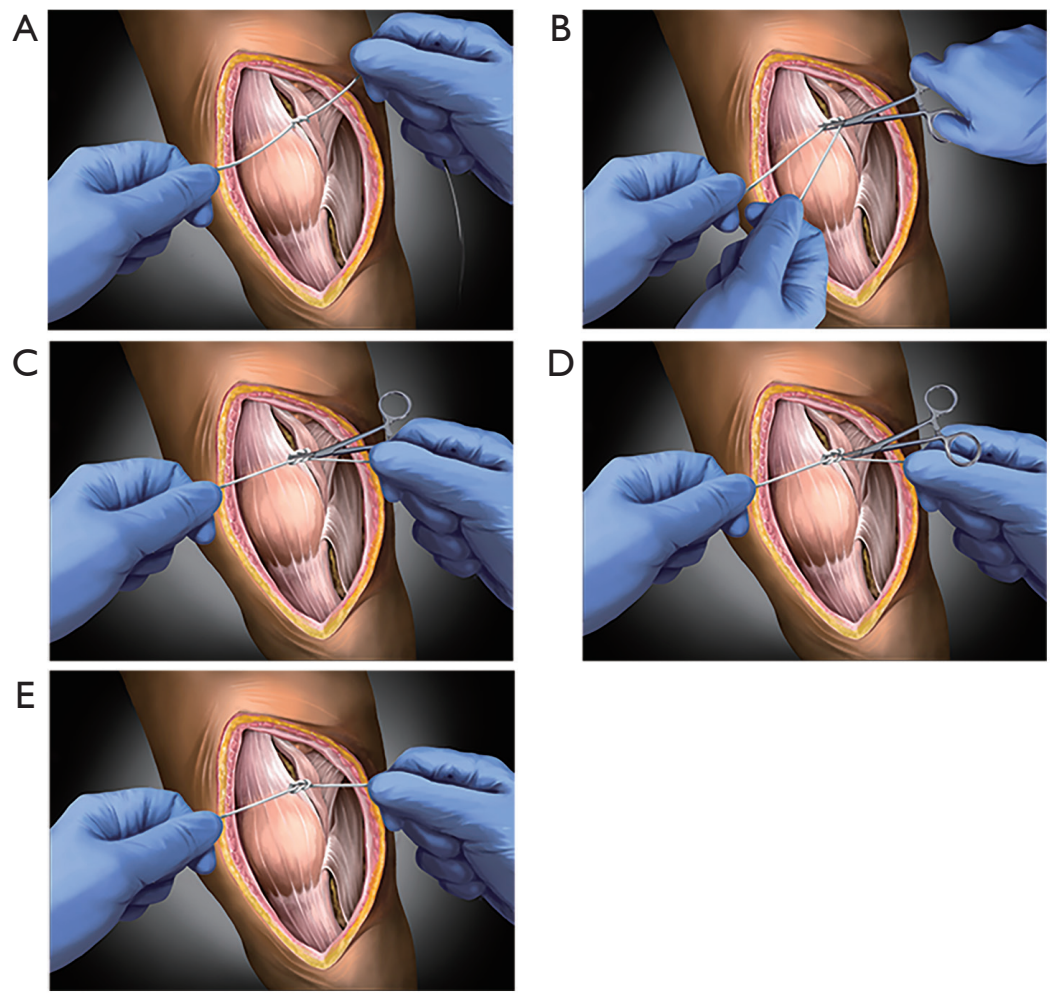

Figure 1 Schematic diagram of the common knotting process with clamp. (A) First knot. (B) Clamp. (C) Second knot. (D) Withdraw clam. (E) Tightened knots.

gap formation when the clamp is removed. Additionally, it is time-consuming and laborious as the assistant clamps the suture when making knots.

For the above reasons, we introduce a novel surgical knotting technique, which we have named the SH-9Hospital knotting technique for suturing soft tissues under tension, such as the joint capsule, fascia, and deep tissues. The SH9Hospital knotting technique has various advantages. This technique allows the surgeon to perform the knot independently without assistance and therefore, it is a timesaving and convenient approach. The SH-9Hospital knotting technique reduces the risk of suture damage by avoiding clamping of the suture and providing a strong tensile load. It is easy to learn this technique to ensure secure and tensional knots for tissues. The main objective of this study was to describe the clinical practice and investigate the security and efficiency of the SH-9Hospital knotting technique.

\section{Methods}

The study was conducted in accordance with the
Declaration of Helsinki (as revised in 2013). The study was approved by Ethics Committee of Shanghai Ninth People's Hospital affiliated to Shanghai Jiao Tong University School of Medicine (No.: SH9H-2020-T396-1) and informed consent was taken from all individual participants.

\section{Knotting methods}

The absorbable braided suture (USP \#1) material consisting of polyglycolic acid (B. Braun Surgical, S.A. Rubi, Spain) was used in this study. The knots were performed by either fixing with a clamp (clamp group) or the SH-9Hospital knotting method (SH-9Hospital group) on the smooth surface of a cylinder. Each group consisted of five suture loops. In the clamp group, the first suture knot was fixed with a clamp by the assistant and then a subsequent reverse knot was performed while the clamp was removed. Three additional reciprocating knots were fastened following the initial square knot (Figure 1). In the SH-9Hospital group, the first suture knot was performed regardless of loosening and then the left forefinger was hooked on to the 
A

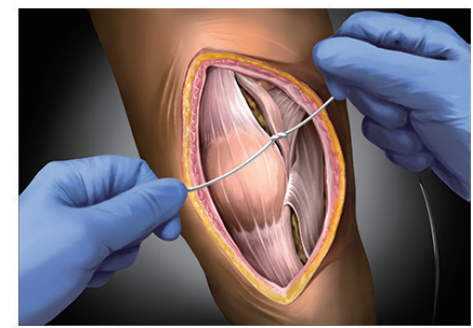

$\mathrm{D}$

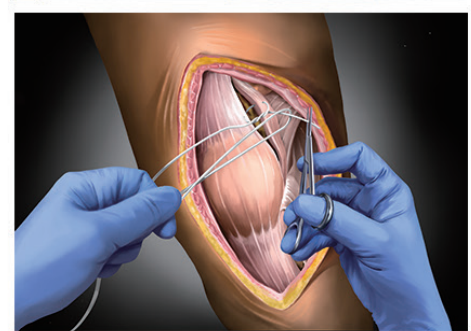

G

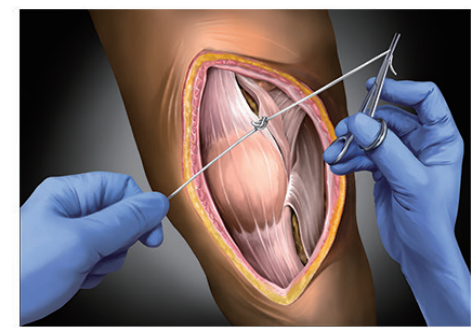

B

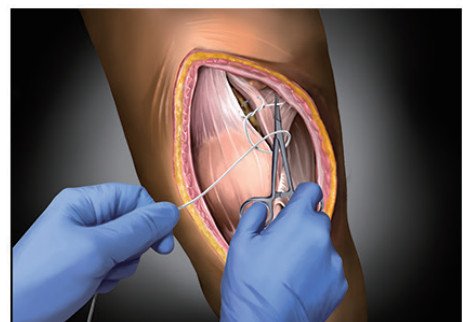

E

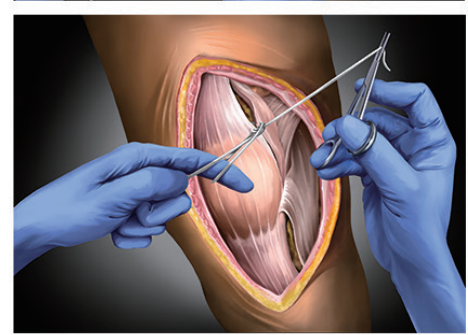

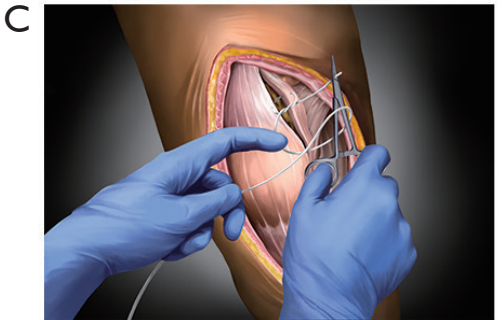

$\mathrm{F}$

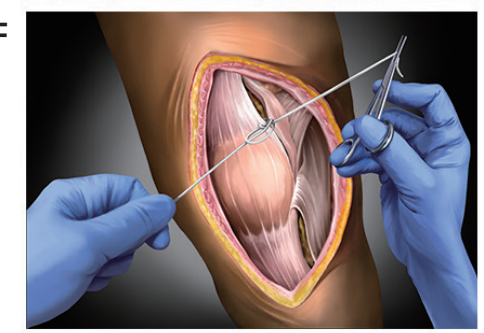

Figure 2 Schematic diagram of the SH-9Hospital knotting technique process. (A) First knot. (B) Instrument tying. (C) One finger hooks the suture. (D) Pulling the suture with finger and instrument. (E) Tightening the suture with finger and instrument. (F) Keeping the Instrument side suture tight and pulling the suture of the finger side. (G) Tightened knots.

suture when a second knot was performed with the needle holder. Tension was applied to the suture until the knot was fastened. While maintaining tension at the end of the suture with the needle holder, the forefinger was removed to tighten the opposite end of the suture in the left hand. Three reciprocating reverse knots were performed (Figure 2). The demonstration video of the SH-9Hospital knotting technique in operation could be seen in Video 1. The knotting time of the two groups was also recorded.

\section{Mechanical testing}

Mechanical testing was performed on the five loops from each group using a universal material testing machine (CMT6103, MTS systems, Eden Prairie, Minnesota, USA). Suture loops were fixed with the jaws of the testing machine. A preload of $5 \mathrm{~N}$ was applied to each specimen to prevent knot relaxation. Loops were loaded at a speed of $20 \mathrm{~mm} / \mathrm{min}$ until the knots broke. A load-elongation curve and ultimate tensile load (UTL) were recorded for each specimen. UTL was defined as suture breakage of the knot.

\section{Statistical analysis}

SPSS software (Version 19; SPSS Inc., Chicago, IL, USA) was used for statistical analysis. Student's $t$-test was used to compare the means of the knot time and UTL. A P value $<0.05$ was considered statistically significant.

\section{Results}

The knotting time measurements of the clamp and $\mathrm{SH}$ 9Hospital methods are shown in Figure $3 \mathrm{~A}$. The mean knotting time \pm standard deviation (SD) in the clamp and SH-9Hosptial groups was $36.40 \pm 1.50 \mathrm{~s}$ (range, 32-41 s) and $24.80 \pm 1.16 \mathrm{~s}$ (range, 21-28 s), respectively. Therefore, the SH-9Hospital knotting technique was faster than the traditional method with the clamp $(\mathrm{P}<0.05)$.

The UTL measurements of the clamp and SH-9Hospital methods are shown in Figure 3B. The mean UTL in the 
A

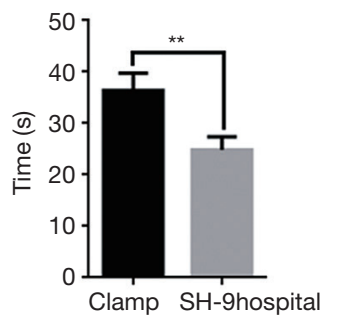

B

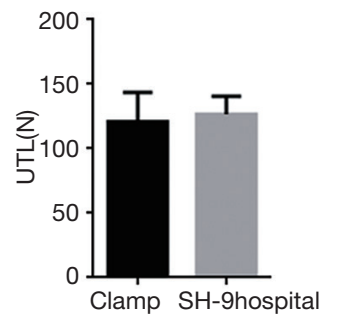

Figure 3 The mean knotting time (A) and mean ultimate tensile load (UTL) (B) in the clamp and SH-9Hosptial groups. ${ }^{* *}, \mathrm{P}<0.01$.

clamp and SH-9Hospital group was $120.8 \pm 10.14 \mathrm{~N}$ (range, $81.11-136.55 \mathrm{~N}$ ) and $126.5 \pm 6.29 \mathrm{~N}$ (range 104.88-139.56 N), respectively. There was no significant difference in the UTL between the two groups $(\mathrm{P}>0.05)$, and therefore, the knot strength of the SH-9Hospital technique was not inferior to the tradition clinical practice with the clamp.

\section{Discussion}

Integrated incision closure, especially deep tissue and capsular closure, is indispensable in orthopaedic operations, which can affect surgical outcomes, lower postoperative complications, promote early rehabilitation, and increase patient satisfaction (9-11). Deep and capsular sutures are often performed with tension (12). The first throw tends to slip before the tying of the second locking loop due to this tension. A common clinical practice is to fix the first knot with a clamp by an assistant and then tie a reverse locking knot (13). However, this is a time-consuming and laborious process for this practice. Moreover, clamp usage may influence the strength of the knots (6). Therefore, we evaluated a novel and efficient surgical knotting technique, the SH-9Hospital knotting technique, which has been used in clinical practice in our hospital and achieved satisfactory and definite consequences.

Operation time is an important factor in the intraoperative safety and postoperative rehabilitation of patients $(9,14)$. A prolonged operational time can increase the risk of surgery and complications $(15,16)$. Based on the results of our study, a faster knotting time with the SH-9Hospital technique was observed compared to the traditional clamp technique. This time that has been saved can potentially be used for other operative procedures performed by the surgeon. In addition, it can decrease the anaesthesia time and potentially decrease anaesthesia- associated complications in patients.

The SH-9Hospital knotting technique is simple, convenient, fast, and easy to learn and perform. The SH9Hospital knots can be performed by the surgeon without any assistance. It is labour-saving because it allows the assistant to perform other routine operating procedures or tasks, which can potentially accelerate the operating process.

When we are confronted with a high-tension closure, the usual practice is clamping the first throw with the help of an assistant. The results of Mehmet's research demonstrated that the ultimate failure load of the monofilament nonabsorbable polypropylene suture was significantly decreased when the first throw was fixed with a clamp to prevent slippage; however, monofilament polyglyconate, nylon, and braided absorbable polyglactin sutures were not affected by fixing the first throw (5). According to Bisson's study, the UTL was reduced in No. 2 Force Fiber suture loops tied with serrated or smooth clamps compared to those tied with no clamps, while the use of clamps did not affect the other six sutures, including Ethibond (No. 2 and 5), Fiberwire (No. 2 and No. 5), Ultrabraid (No. 2), and Orthocord (No. 2) (4). In our study, we used an absorbable braided suture made with polyglycolic acid to make the knots. The surgeon could accomplish the SH-9Hospital knots without clamping the first knot to prevent slippage during the tying process and does not need to worry about clamp-associated suture damage. A triple throw is made in the present knotting technique and can achieve a firm lock. The UTL of the SH-9Hospital technique was not inferior to the tradition clamp method.

Our study has several limitations. First, only one size of multifilament absorbable braided suture material was used in our study. Unabsorbable or monofilament sutures and other suture sizes were not tested. Second, the cyclical load was not applied in our study, which would have been more similar to clinical practice because patients are often encouraged to perform early mobilization and cyclic motion of the knee after knee arthroplasty. Finally, our study was conducted in an open and dry environment of the laboratory. The aqueous environment, which can be used to simulate the physiological and pathological environment in patients, was not applied when tying or testing the load of knots.

In conclusion, we presented a novel and efficient surgical knotting technique named the SH-9Hospital for closing high-tension incisions. The SH-9Hospital knotting technique is secure, convenient, and easy to perform. It has 
good strength and reliability for closure of high-tension incisions without knot slippage or suture breakage.

\section{Acknowledgments}

Funding: This study was supported by the Science \& Technology Innovation Fund of Shanghai Ninth People's Hospital, Shanghai Jiaotong University School of Medicine (CK2019002); Shanghai Clinical Medical Center (2017ZZ01023); Shanghai Municipal Key Clinical Specialty (shslczdzk00402, shslczdzk07001); The Youth Doctor Collaborative Innovation Team Project of Shanghai Ninth People's Hospital, Shanghai Jiaotong University School of Medicine (QC201903).

\section{Footnote}

Data Sharing Statement: Available at http://dx.doi. org/10.21037/atm-20-6332

Peer Review File: Available at http://dx.doi.org/10.21037/ atm-20-6332

Conflicts of Interest: All authors have completed the ICMJE uniform disclosure form (available at http://dx.doi. org/10.21037/atm-20-6332). The authors have no conflicts of interest to declare.

Ethical Statement: The authors are accountable for all aspects of the work in ensuring that questions related to the accuracy or integrity of any part of the work are appropriately investigated and resolved. The study was conducted in accordance with the Declaration of Helsinki (as revised in 2013). The study was approved by Ethics Committee of Shanghai Ninth People's Hospital affiliated to Shanghai Jiao Tong University School of Medicine (No.: SH9H-2020-T396-1) and informed consent was taken from all individual participants.

Open Access Statement: This is an Open Access article distributed in accordance with the Creative Commons Attribution-NonCommercial-NoDerivs 4.0 International License (CC BY-NC-ND 4.0), which permits the noncommercial replication and distribution of the article with the strict proviso that no changes or edits are made and the original work is properly cited (including links to both the formal publication through the relevant DOI and the license). See: https://creativecommons.org/licenses/by-nc-nd/4.0/.

\section{References}

1. Kobayashi S, Niki Y, Harato K, et al. The effects of barbed suture on watertightness after knee arthrotomy closure: a cadaveric study. J Orthop Surg Res 2018;13:323.

2. Nett M, Avelar R, Sheehan M, et al. Water-tight knee arthrotomy closure: comparison of a novel single bidirectional barbed self-retaining running suture versus conventional interrupted sutures. J Knee Surg 2011;24:55-9.

3. Zhao C, Hsu CC, Moriya T, et al. Beyond the square knot: a novel knotting technique for surgical use. J Bone Joint Surg Am 2013;95:1020-7.

4. Bisson LJ, Sobel AD, Godfrey D. Effects of using a surgical clamp to hold tension while tying knots with commonly used orthopedic sutures. Knee Surg Sports Traumatol Arthrosc 2012;20:1673-80.

5. Turker M, Yalcinozan M, Cirpar M, et al. Clamp fixation to prevent unfolding of a suture knot decreases tensile strength of polypropylene sutures. Knee Surg Sports Traumatol Arthrosc 2012;20:2602-5.

6. Dobrin PB. Surgical manipulation and the tensile strength of polypropylene sutures. Arch Surg 1989;124:665-8.

7. Hirano Y, Ishikawa N, Watanabe G. Suture damage after grasping with EndoWrist of the da Vinci Surgical System. Minim Invasive Ther Allied Technol 2010;19:203-6.

8. Mulon PY, Zhim F, Yahia LH, et al. The effect of six knotting methods on the biomechanical properties of three large diameter absorbable suture materials. Vet Surg 2010;39:561-5.

9. Krebs VE, Elmallah RK, Khlopas A, et al. Wound Closure Techniques for Total Knee Arthroplasty: An Evidence-Based Review of the Literature. J Arthroplasty 2018;33:633-8.

10. Elmarsafi T, Pierre AJ, Wang K, et al. The vertical contour calcanectomy: an alternative surgical technique to the conventional partial calcanectomy. J Foot Ankle Surg 2019;58:381-6.

11. Patel RM, Cayo M, Patel A, et al. Wound complications in joint arthroplasty: comparing traditional and modern methods of skin closure. Orthopedics 2012;35:e641-6.

12. Barber FA, Herbert MA, Beavis RC. Cyclic load and failure behavior of arthroscopic knots and high strength sutures. Arthroscopy 2009;25:192-9.

13. Huber DJ, Egger EL, James SP. The effect of knotting method on the structural properties of large diameter nonabsorbable monofilament sutures. Vet Surg 1999;28:260-7. 
14. Peersman G, Laskin R, Davis J, et al. Prolonged operative time correlates with increased infection rate after total knee arthroplasty. HSS J 2006;2:70-2.

15. Gowd AK, Liu JN, Bohl DD, et al. Operative time as an independent and modifiable risk factor for short-

Cite this article as: Chang $\mathrm{Y}$, Yu D, Wang L, Zhang J, Yan M, Li H, Mao Y, Zhai Z. A novel and efficient surgical knotting technique for high-tension closures. Ann Transl Med 2021;9(5):375. doi: 10.21037/atm-20-6332 term complications after knee arthroscopy. Arthroscopy 2019;35:2089-98.

16. Bohl DD, Ondeck NT, Darrith B, et al. Impact of Operative Time on Adverse Events Following Primary Total Joint Arthroplasty. J Arthroplasty 2018;33:2256-62.e4. 